\title{
Sesquiterpene Synthase from the Botrydial Biosynthetic Gene Cluster of the Phytopathogen Botrytis cinerea
}

\author{
Cristina Pinedo ${ }^{\dagger, \uparrow}$, Chieh-Mei Wang ${ }^{\ddagger, \uparrow}$, Jean-Marc Pradier ${ }^{\S}$, Bérengère Dalmais ${ }^{\S}$, Mathias Choquer ${ }^{\S}$, \\ Pascal Le Pêcheur ${ }^{\S}$, Guillaume Morgant ${ }^{\S}$, Isidro G. Collado ${ }^{\dagger, *}$, David E. Cane ${ }^{\ddagger, *}$, and Muriel Viaud ${ }^{\S, *}$ \\ †Departamento de Química Orgánica, Facultad de Ciencias, Universidad de Cádiz, 11510 Puerto Real, Spain, ${ }^{\ddagger}$ Department \\ of Chemistry, Box H, Brown University, Providence, Rhode Island 02912-9108, and §UMR BIOGER, INRA, Route de Saint- \\ Cyr, 78086 Versailles, France. ๆThese authors contributed equally to this work.
}

$\mathbf{F}$ ilamentous fungi produce a wide variety of medically and agriculturally important secondary metabolites. Although the native biological role of many of these naturally occurring compounds may often remain unclear, relationships with fungal development, pathogenicity, chemical defense, and fitness have been established (1). For example, the well-known mycotoxic trichothecenes are fungal sesquiterpenes that have important consequences for both human and animal health, while the fungal sesquiterpene abscisic acid and the diterpene gibberellins can act as plant hormones. The key enzymes for the biosynthetic pathways leading to each of these metabolites are the sesquiterpene and diterpene synthases that catalyze the carbocationic cyclization of the universal precursors farnesyl diphosphate and geranylgeranyl diphosphate, respectively. The genes encoding microbial terpene cyclases are usually part of a physical cluster of genes whose encoded protein products together catalyze all or most of the steps in the biosynthetic pathway leading to the final terpene metabolite $(2,3)$.

Botrytis cinerea is the causal agent of the economically important gray mold disease that affects more than 200 ornamental and agricultural plant host species (4, 5). In contrast to its usual deleterious role, infection of Sémillon grapes with $B$. cinerea under specific climatic conditions also plays a vital role in the production of Sauternes and other botrytised dessert wines, leading to the designation "la pourriture noble" or "noble rot" (6). As a necrotrophic and polyphageous pathogen, the gray mold fungus secretes nonspecific phytotoxins that kill cells from a large spectrum of plants. Among the
ABSTRACT The fungus Botrytis cinerea is the causal agent of the economically important gray mold disease that affects more than 200 ornamental and agriculturally important plant species. $B$. cinerea is a necrotrophic plant pathogen that secretes nonspecific phytotoxins, including the sesquiterpene botrydial and the polyketide botcinic acid. The region surrounding the previously characterized $B C B O T 1$ gene has now been identified as the botrydial biosynthetic gene cluster. Five genes including $B C B O T 1$ and $B C B O T 2$ were shown by quantitative reverse transcription-PCR to be co-regulated through the calcineurin signaling pathway. Inactivation of the $B C B O T 2$ gene, encoding a putative sesquiterpene cyclase, abolished botrydial biosynthesis, which could be restored by in trans complementation. Inactivation of $B C B O T 2$ also resulted in overproduction of botcinic acid that was observed to be strain-dependent. Recombinant ВсВОТ2 protein converted farnesyl diphosphate to the parent sesquiterpene of the botrydial biosynthetic pathway, the tricyclic alcohol presilphiperfolan-8 $\beta$-ol.

*Corresponding authors, isidro.gonzalez@uca.es, david_cane@brown.edu, viaud@versailles.inra.fr

Received for review September 14, 2008 and accepted November 4, 2008.

Published online November 26, 2008

10.1021/cb800225v CCC: $\$ 40.75$

(C) 2008 American Chemical Society 


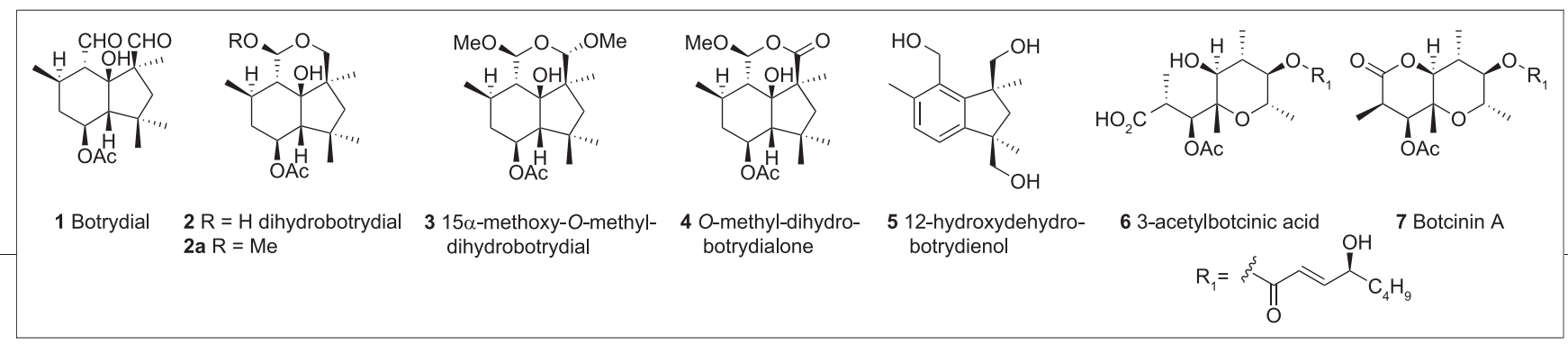

Figure 1. Metabolites isolated from $B$. cinerea strains and mutants.

numerous metabolites isolated from fermentation broths ( 7 ), the most well-known are the bicyclic sesquiterpene botrydial (1) and several related compounds with lower phytotoxic potential (Figure 1) (8). Botrydial, which is produced during plant infection, induces chlorosis and cell collapse (9). In addition to this toxic sesquiterpene, aggressive strains of $B$. cinerea also produce polyketides such as botcinic acid and related botcinins that display phytotoxic and antifungal activity (Figure 1) (10). We have recently shown that the BCBOT1 (for Botrytiscinerea BOTrydial biosynthesis) gene, which encodes a P450 monooxygenase, is responsible for one of the final steps of botrydial biosynthesis (Figure 2, panel a) (11). Indeed, bcbot1 $\Delta$ deletion mutants were unable to produce either $10 \beta, 15 \alpha$-dihydroxyprobotryane (8), botrydial (1), or dihydrobotrydial (2) (Figure 1 and Figure 2, panel a). Inactivation of the BCBOT1 gene in three different strains demonstrated that botrydial is a strain-dependent virulence factor, with some strains also producing botcinic acids as virulence agents.

The sequencing of the genomes of the B05-10 and T4 strains of $B$. cinerea at the Broad Institute and the Genoscope, respectively (12), has provided the opportunity to further characterize the BCBOT1 gene locus. Our previous study indicated that this gene is surrounded by two other putative P450 monooxygenase-encoding genes (BCBOT3 and BCBOT4), as well as the $B C B O T 2$ gene, encoding a probable sesquiterpene synthase
(Figure 3, panel a) (11). The data set from the B. cinerea T4 strain (http://www.genoscope.cns.fr/D provided a 16-kb DNA contig containing this region. Using data from Expressed Sequence Tags (13), we have confirmed the cDNA sequences of each of the four previously identified genes along with that of a fifth expressed gene, $B C B O T 5$, located at the $5^{\prime}$-end of the cluster (Figure 3, panel a). The deduced amino acid sequence for this fifth gene showed high homology to known acetyl transferases, suggesting that it is likely responsible for the introduction of the characteristic acetyl group of botrydial (1) and related late-stage biosynthetic intermediates (Figure 1).

\section{RESULTS AND DISCUSSION}

\section{BCBOT2 Gene Is Predicted To Encode a Terpene}

Synthase. The predicted 399 amino acid sequence of the $\mathrm{BcBOT} 2$ protein shows significant levels of similarity to those of microbial terpene synthases. Most importantly, the BсВОT2 protein has both of the two strictly conserved active-site motifs that are essential to binding of the three $\mathrm{Mg}^{2+}$ cations that constitute the required cofactor for all terpene synthase reactions: an aspartate-rich sequence, DDQFD, that is found at amino acid 141 and an "N(S/T)(E/D)" triad, NDVLSYRKD, located 144 amino acids downstream of the DDXXD motif (14). Thus the BCBOT2 gene product would be expected to correspond to the key sesquiterpene syn- a

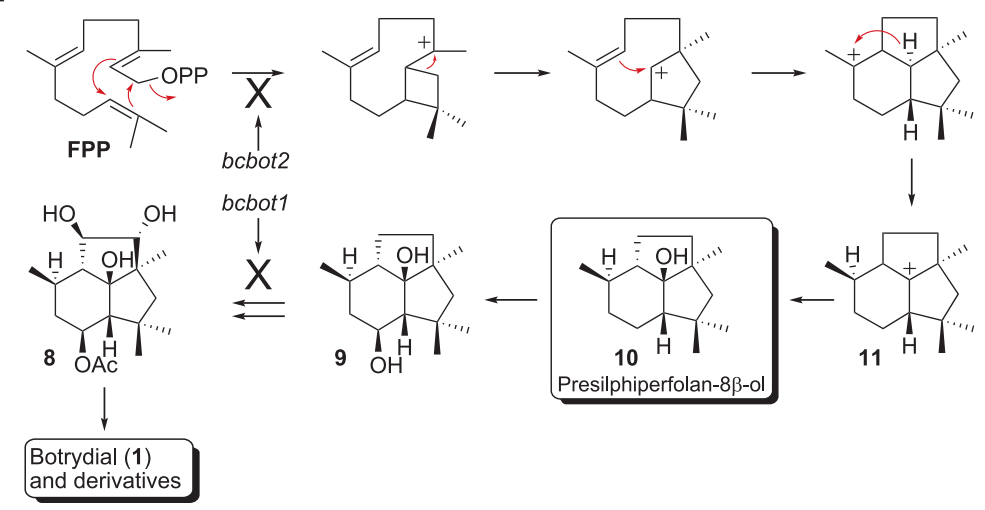

b

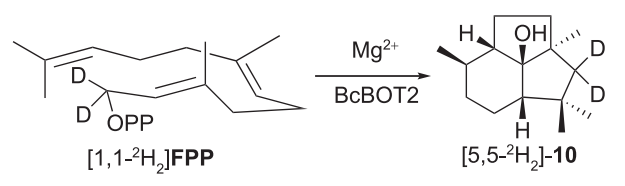

Figure 2. Biosynthesis of botrydial by Botrytis cinerea. a) Cyclization of farnesyl diphosphate (FPP) to presilphiperfolan-8 $\beta$-ol (10) catalyzed by the BCBOT2 protein and biosynthesis of botrydial from 10. B. cinerea mutant bcbot1 does not form botrydial and other late-stage intermediates but does accumulate 9 and other probotryanes, whereas mutant bcbot2 is blocked in the entire pathway. b) Cyclization of $\left[1,1-{ }^{2} \mathrm{H}_{2}\right]$ farnesyl diphosphate to $\left[5,5-{ }^{2} \mathrm{H}_{2}\right]$ presilphiperfolan-8 $\beta$-ol. 


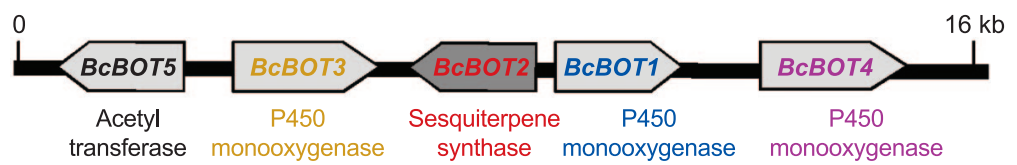

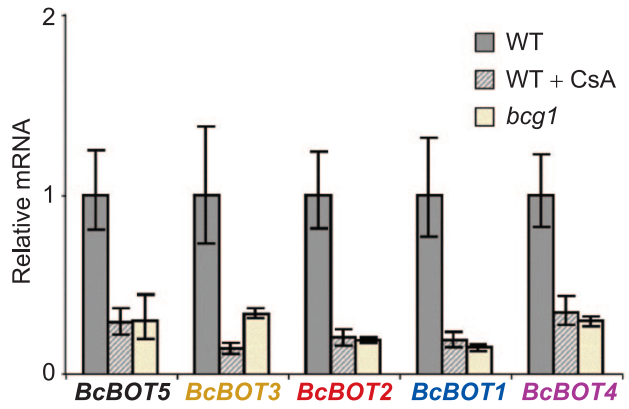

Figure 3. Physical cluster of co-regulated genes of Botrytis cinerea flanking the botrydial biosynthesis gene BCBOT1. a) Five open reading frames of the botrydial biosynthetic gene cluster (NCBI accession number AY277723). Putative functions predicted by protein sequence similarity are indicated under each gene. b) Down-regulation of the clustered genes in the presence of the calcineurin inhibitor cyclosporin $A\left(10 \mu g \mathrm{~mL}^{-1}\right)$ and in the bcg1 $\Delta$ mutant (18). The expression level of each of the five clustered genes was analyzed by qRT-PCR with the constitutively expressed elongation factor gene EF1b serving as a control (35).

thase responsible for catalyzing the cyclization of farnesyl diphosphate.

B. cinerea bcbot2s Deletion Mutants Are Blocked in the First Step of Botrydial Biosynthesis. To test this hypothesis, we first used a gene inactivation approach based on homologous recombination. The knockout plasmid $\mathrm{p} B C B O T 2 \Delta$, harboring the $B A R$ gene that confers resistance to bialaphos in place of the $B C B O T 2$ gene, was initially used to transform protoplasts of the B. cinerea B05-10 and T4 strains, but no bcbot2 $\Delta$ deletion mutant could be isolated from a total of 30 transformants. Gene targeting in fungi was recently shown to be facilitated by the construction of $k u$ recipient strains that are altered in the Non-Homologous-end-Joining DNA repair system and therefore show a high level of homologous recombination (15). The recipient strains

TABLE 1. Metabolites identified in Botrytis cinerea wild-type strains, recipient strains (ku mutants), and bcbot2 mutants ${ }^{a}$

\begin{tabular}{|c|c|c|c|c|c|c|c|}
\hline \multirow{2}{*}{$\frac{\text { Strain or mutant }}{\text { B05-10 wild-type }}$} & & \multicolumn{4}{|c|}{ Botryanes $\left(\mathrm{mg} \mathrm{L}^{-1}\right)$} & \multicolumn{2}{|c|}{ Botcinic acid and derivatives $\left(\mathrm{mg} \mathrm{L}^{-1}\right)$} \\
\hline & & 1 & 2 & $2 a$ & 3 & 7 & \\
\hline & & 2 & 1 & 1.1 & 1.7 & 0.17 & \\
\hline \multirow{2}{*}{\multicolumn{2}{|c|}{ B05-10 ku70s }} & 1 & 2 & $2 a$ & 3 & 7 & \\
\hline & & 2 & 0.7 & 1 & 1.3 & 0.2 & \\
\hline \multirow{2}{*}{\multicolumn{2}{|c|}{ B05-10 ku70 $\Delta$ bcbot $2 \Delta-6$}} & \multirow{2}{*}{\multicolumn{4}{|c|}{ none }} & 6 & 7 \\
\hline & & & & & & 57 & 3 \\
\hline \multirow{2}{*}{\multicolumn{2}{|c|}{ B05-10 ku70 $\Delta$ bcbot2 $\Delta$ BсBOT2 }} & 1 & 2 & $2 a$ & 3 & 7 & \\
\hline & & 1.7 & 0.7 & 1 & 2 & 0.2 & \\
\hline Strain or mutant & & \multicolumn{4}{|c|}{ Botryanes $\left(\mathrm{mg} \mathrm{L}^{-1}\right)$} & \multicolumn{2}{|c|}{ Botcinic acid and derivatives $\left(\mathrm{m} \mathrm{L}^{-1}\right)$} \\
\hline \multirow[t]{2}{*}{ T4 wild-type } & 2 & & 4 & & 5 & \multicolumn{2}{|l|}{ none } \\
\hline & 1.7 & & 33 & & 1 & & \\
\hline \multirow[t]{2}{*}{ T4 ku80s } & 2 & & 4 & & 5 & none & \\
\hline & 2.3 & & 30 & & 1.7 & & \\
\hline T4 ku80s bcbot2s & & & none & & & none & \\
\hline
\end{tabular}

${ }^{a}$ Numbers correspond to compounds in Figure 1. 


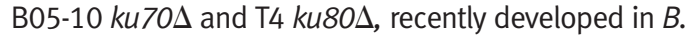
cinerea (16), were therefore transformed with the plasmid $\mathrm{pBCBOT} 2 \Delta$, thereby yielding two bcbot $2 \Delta$ deletion mutants within the B05-10 background (nos. 6 and 12) and one such mutant in the T4 background (no. 3) out of a total of 10 analyzed transformants. The metabolites produced by the wild-type $B$. cinerea strains and by the corresponding $B C B O T 2 \triangle$ mutants were each identified by high-pressure liquid chromatography (HPLC) comparison with authentic samples, as well as ${ }^{1} \mathrm{H}$ and ${ }^{13} \mathrm{C}$ NMR (Figure 1 and Table 1). The wild-type B05-10 strain and the derived $k u \Delta$ recipient strain produced both botrydial (1) and its derivatives ( 2 and $\mathbf{3}$ ), as well as small amounts of botcinin A (7). As expected (11), both the wild-type $\mathrm{T} 4 \mathrm{strain}$ and the derived $k u \Delta$ recipient strain produced botrydial but neither botcinin A nor any other botcinic acid derivative. Significantly, neither of the bcbot $2 \Delta$ null mutants generated from either the parent B05-10 or the T4 backgrounds was able to produce either botrydial or any of its derivatives and biosynthetic intermediates. These data established that the protein encoded by $B C B O T 2$ is required for the first step of botrydial biosynthesis, the presumptive cyclization of FPP to the key probotryane alcohol intermediate, presilphiperfolan-8 $\beta$-ol (10) (Figure 2, panel a). Interestingly, inactivation of the BCBOT2 gene in the B05-10 background also led to an apparent redirection of metabolism, resulting in a significant overproduction of both botcinic acid (6) (57 $\left.\mathrm{mg} \mathrm{L}^{-1}\right)$ and botcinin A (7) ( $3 \mathrm{mg} \mathrm{L}^{-1}$ ), compared to the minimal level of $<0.2 \mathrm{mg} \mathrm{L}^{-1}$ of 7 in the wild-type $B$. cinerea B05-10 parent strain and the absence of 6 altogether. (Table 1 ). By contrast, neither of these latter botcinate derivatives was detected in the corresponding B. cinerea T4 ku80D bcbot $2 \Delta$ mutant.

To validate the inferred function of the $\mathrm{BcBOT} 2$ protein, we reintroduced a copy of the native $B C B O T 2$ gene into the B05-10 ku70 b bcbot2s-6 null mutant.

Southern blot analysis showed that the integration occurred at the targeted locus, resulting in a tandem pattern with the knockout copy of the gene. The metabolite profile of the complemented transformant indicated that the biosynthesis of botrydial and its derivatives had been restored (Table 1), while interestingly the production of botcinic acid and its derivatives reverted to the original low levels characteristic of the parent B05-10 strain. These data demonstrate that the BCBOT2 gene encodes the enzyme responsible for the committed step of botrydial biosynthesis, proposed to correspond to the cyclization of FPP.

Five Contiguous Genes of the Botrydial Biosynthetic Cluster Are Co-regulated by a Common Pathway. The $B C B O T 2$ gene is adjacent to the $B C B O T 1$ gene that has previously been shown to encode a P450 monooxygenase required for botrydial biosynthesis (11) (Figure 3, panel a). The combined evidence that two neighboring genes, $B C B O T 1$ and $B C B O T 2$, are involved in different steps of the toxin synthesis thereby validates the designation of the genomic locus as the botrydial biosynthetic gene cluster. Surrounding the BCBOT1 and $B C B O T 2$ loci are three additional co-regulated genes that code for enzymes with putative functions in the biosynthesis of botrydial (Figure 3, panel a). Macro-array analyses have previously shown that the three central genes $B C B O T 3, B C B O T 2$ and $B C B O T 1$ (previously known as CND11, CND15 and CND5 for CalciNeurinDependent) are co-regulated by a transduction pathway that depends on both the G $\alpha$ protein BCG1 and calcineurin $(17,18)$. To evaluate whether the two flanking genes BCBOT4 and BCBOT5 are also co-regulated through this same pathway, we used quantitative reverse transcription-PCR (qRT-PCR) to analyze expression of all five genes of the cluster in both wild-type B. cinerea $\mathrm{B} 05-10$ and a derived bcg1D mutant from which the gene for the BCG1 protein had been deleted (19). The qRT-PCR results (Figure 3, panel b) indicated that all five clustered genes are significantly downregulated both by the calcineurin inhibitor cyclosporin $A$ and by inactivation of the $B C G 1$ gene, thus clearly demonstrating that these five contiguous genes are coregulated by the same BCG1-calcineurin transduction pathway (18). In this regulatory pathway, the external signal is known to be relayed successively through the $G \alpha$ protein BCG1, phospholipase $C$, calcineurin phosphatase, and CRZ1, a $\mathrm{Cys}_{2} \mathrm{His}_{2}$ zinc finger transcription factor (20). The gene promoters of the botrydial biosynthetic cluster are therefore presumed to be activated directly by the global transcription factor CRZ1 or indirectly by a CRZ1-dependent transcription factor. This putative specific transcription factor might then be of the $\mathrm{Zn}(\mathrm{II})_{2} \mathrm{Cys}_{6}$ class, as commonly observed in gene clusters for secondary metabolism (3). Both the $5^{\prime}$ - and $3^{\prime}$-ends of the 16-kb botrydial cluster are TA-rich (>80\%) which has made difficult the cloning and sequencing of the surrounding genomic sequences and the identification of potential additional $B C B O T$ genes. 
a

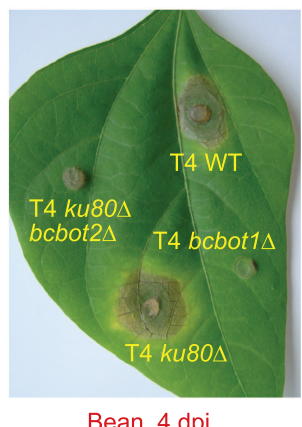

b

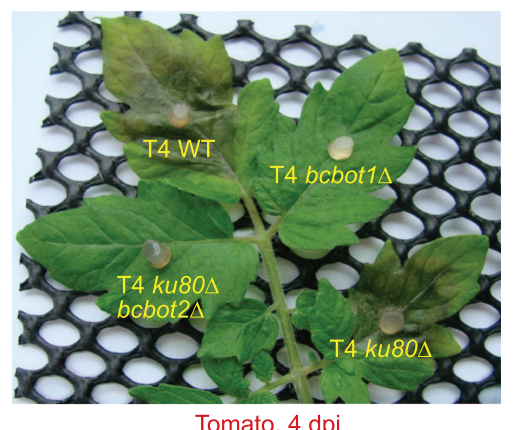

C

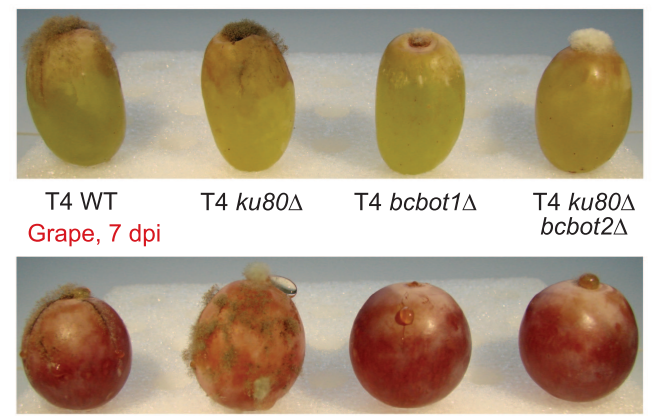

Figure 4. Pathogenicity assays of Botrytis cinerea. Wild-type T4 strains, recipient strains (ku mutants), and botrydial mutants on a) bean leaves, b) tomato leaves, and c) grape berries. Leaves were inoculated with plugs of mycelium, and the tops of the berries were inoculated with $10^{3}$ conidia.

Although the minimal set of enzymes required for the biosynthesis of botrydial is apparently present in this five-gene biosynthetic cluster, additional enzymes, a transporter, or a specific transcription factor may also be necessary for production of botrydial by $B$. cinerea.

Botrydial Is a Strain-Dependent Virulence Factor. Neither saprophytic growth nor conidiation of the bcbot2s mutants raised on either standard culture (NY) or vegetable-rich (V8) media were affected compared to the corresponding wild-type strains of $B$. cinerea. Our previous studies with the botrydial non-producing mutant, $b c b o t 1 \Delta$, had suggested, however, that the sesquiterpene toxin must be regarded as a strain-dependent virulence factor. Indeed inactivation of the $B C B O T 1$ gene had led to reduced virulence by the T4 recipient strain but not in the more virulent strain SAS56 (11).

The availability of the bcbot $2 \Delta$ mutants allowed investigation of the role of botrydial in the highly virulent model B. cinerea B05-10 strain. Standard virulence assays performed by inoculating young mycelium (Table 2) or conidia onto bean and tomato leaves gave similar results. Lesions caused by $B$. cinerea B05-10 ku70 $\Delta$ bcbot $2 \Delta$ mutants were similar to those due to wild-type, suggesting that botrydial is not essential for infection in this genetic background. In contrast, the virulence of the $B$. cinerea $\mathrm{T} 4 \mathrm{ku} 80 \Delta$ bcbot $2 \Delta$ mutant was reduced by an amount similar to that of the previously described T4 bcbot1 $\Delta$ mutant (11) (Figure 4 and Table 2). The

\begin{tabular}{|c|c|c|}
\hline B. cinerea strain & $\begin{array}{l}\text { Bean leaves, } \\
\text { lesion size } 5 \mathrm{dpi}^{b} \\
(\mathrm{~mm})\end{array}$ & $\begin{array}{l}\text { Tomato leaves, } \\
\text { lesion size } 4 \mathrm{dpi} \\
(\mathrm{mm})\end{array}$ \\
\hline B05-10 WT & $30.9 \pm 4.5$ & $35.1 \pm 3.8$ \\
\hline B05-10 ku70s & $29.5 \pm 5.1$ & $36.1 \pm 5.2$ \\
\hline B05-10 ku $70 \Delta$ bcbot $2 \Delta-6$ & $29.5 \pm 5.3$ & $35.8 \pm 3.4$ \\
\hline B05-10 ku70 b bcbot $2 \Delta-12$ & $28.6 \pm 4.6$ & $35.8 \pm 3.6$ \\
\hline B05-10 ku70 $\Delta$ bcbot $2 \Delta$ BcBOT2 & $31.7 \pm 3.1$ & $33.0 \pm 3.6$ \\
\hline T4 WT & $22.7 \pm 5.0$ & $28.5 \pm 4.9$ \\
\hline T4 ku80s & $21.6 \pm 3.7$ & $25.0 \pm 5.3$ \\
\hline T4 ku80 $\Delta$ bcbot $2 \Delta$ & $2.8 \pm 4.1$ & $3.9 \pm 7.2$ \\
\hline T4 bcbot1s & $6.1 \pm 4.8$ & $7.5 \pm 7.9$ \\
\hline
\end{tabular}

${ }^{a}$ Values are means and standard deviation of at least 15 lesions resulting from 3 independent assays with $5-7$ independent leaves per strain or mutant. ${ }^{b} \mathrm{dpi}=$ days post-inoculation. 
botrydial-defective T4 mutants were still able to penetrate the plant tissues, but the infection was arrested in the early stages of colonization. This in planta growth defect of the $B$. cinerea T4 ku80 $\Delta$ bcbot $2 \Delta$ mutant was also observed on grapevine berries inoculated with conidial suspensions (Figure 4, panel c and Table 2). Taken together, these data indicated that botrydial is necessary for colonization of plant tissue by the $B$. cinerea $\mathrm{T} 4$ strain but not by the more virulent B05-10 strain.

As a necrotrophic pathogen, $B$. cinerea induces host cell death as it colonizes plant tissue (5). Among the probable effectors are phytotoxic secondary metabolites such as botrydial and botcinic acid derivatives whose specific modes of action are still unclear. The properties of the botrydial non-producing bcbot $2 \Delta$ mutant have confirmed that the sesquiterpene toxin is a strain-dependent virulence factor, consistent with the results previously obtained with the bcbot $1 \Delta$ mutant (11). Thus despite the inability of the bcbot1 $\Delta$ and $b c b o t 2 \Delta$ mutants of highly aggressive strains such as B. cinerea SAS56 and B05-10 to produce botrydial, they still retain substantial virulence on all of the tested hosts. Botcinic acid derivatives are overproduced in the fully virulent $B$. cinerea B05-10 bcbot $2 \Delta$ mutants, whereas botcinates are completely absent in the T4 bcbot $2 \Delta$ mutant, which exhibits a defect in colonization of plant tissues. Botrydial and botcinic acid derivatives may therefore have a redundant function in highly virulent strains whereby botcinic acids are able to compensate for the absence of botrydial by an unknown regulatory mechanism. One hypothesis would be feedback control by botrydial on the signal transduction pathways regulating synthesis of both toxins, but such mechanisms have not been described so far in fungi. Expression analysis of the $B$. cinerea genes encoding polyketide synthases are in progress to identify botcinic acid biosynthetic genes and to address these questions.

ВсВ0т2 Protein Is a Sesquiterpene Synthase. We have also directly established the biochemical function of the protein encoded by the $B C B O T 2$ protein. Following heterologous bacterial expression in Escherichia coli of a 1.2-kb synthetic gene corresponding to $B C B O T 2$ with codons optimized for $E$. coli, the soluble BcBOT2 protein, carrying an $\mathrm{N}$-terminal $\mathrm{His}_{6}$ tag, was purified to $>95 \%$ homogeneity by metal affinity Ni-NTA chromatography. The recombinant BcBOT2 protein was shown to have the expected molecular mass by MALDI-TOF mass spectrometry. GC-MS analysis of the products resulting from the incubation of farnesyl diphosphate (FPP) with recombinant $\mathrm{BCBOT} 2$ protein revealed the formation of the predicted sesquiterpene alcohol, presilphiperfolan8 $\beta$-ol (10, $\left.\mathrm{C}_{15} \mathrm{H}_{26} \mathrm{O}, \mathrm{m} / \mathrm{z} 222\right)$, as the major (>95\%) product, which was shown to be identical with an authentic sample of 10 by direct comparison of GC retention times on both achiral and chiral capillary columns, as well as by mass spectra (Supplementary Figure 2) (21). Preparative-scale incubation with FPP yielded a sample of 10 whose ${ }^{1} \mathrm{H}$ NMR spectrum was identical with that of authentic 10 (Supplementary Figure 3). Similar incubation of $\left[1,1-{ }^{2} \mathrm{H}_{2}\right] \mathrm{FPP}$ with recombinant $\mathrm{BcBOT} 2$ protein gave $\left[5,5^{-2} \mathrm{H}_{2}\right]-10\left(\mathrm{C}_{15} \mathrm{H}_{22} \mathrm{D}_{2}, m / z 224\right)$ (Figure 2, panel b), whose ${ }^{1} \mathrm{H}$ NMR spectrum was identical to that of unlabeled 10 except for the absence of the characteristic $1 \mathrm{H}$ doublets $(V=12 \mathrm{~Hz})$ at $\delta 2.25$ and 1.30 corresponding to each of the geminal $\mathrm{H}-5$ methylene protons (Supplementary Figures 4 and 5). Using $\left[1-{ }^{3} \mathrm{H}\right] \mathrm{FPP}$ as substrate, the reaction exhibited typical Michaelis-Menten kinetics (Supplementary Figure 6), with a turnover number $k_{\text {cat }}$ of $3.23 \pm 0.15 \mathrm{~s}^{-1}$ and Michaelis constant $K_{\mathrm{m}}$ for FPP of $6.04 \pm 0.98 \mu \mathrm{M}$, comparable to typical steady-state kinetic parameters for other microbial terpene synthases $(14,22)$.

Cyclization Mechanism. Incorporation experiments with isotopically labeled precursors using intact cultures of $B$. cinerea have previously established the origin of the carbon skeleton of botrydial, as well as the operation of a 1,3-hydride shift during the cyclization of FPP, leading to a proposal for the biosynthesis of botrydial $(7,23)$ that is now reinforced by the demonstrated enzymatic conversion of FPP to presilphiperfolan- $8 \beta-0 l$ 10 (21) (Figure 2, panel b). The finding that recombinant $\mathrm{BcBOT} 2$ protein catalyzes the cyclization of FPP to the sesquiterpene alcohol presilphiperfolan-8 $\beta$-ol (10), combined with the observation that the corresponding $B C B O T 2$ gene is essential to the formation of botryane and probotryane sesquiterpenes, firmly establishes the biochemical function of this gene, which is thus shown to be responsible for the first committed step in the biosynthesis of botrydial.

Although several probotryane metabolites such as $\mathbf{8}$, presumptive intermediates of botrydial biosynthesis, have previously been isolated along with the phytotoxin botrydial (1) and its derivatives $\mathbf{2}-\mathbf{5}$ from the fungal plant pathogen $B$. cinerea $(7)$, the parent sesquiterpene alcohol presilphiperfolan-8 $\beta$-ol (10) has never been isolated from $B$. cinerea itself. The tricyclic alcohol $\mathbf{1 0}$ and 

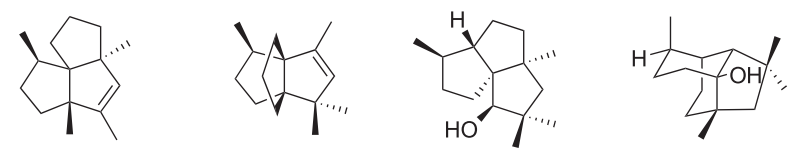

Isocomene

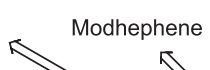

Cameroonan-7-ol

Prenopsan-8-ol

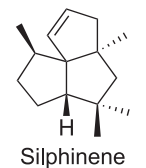

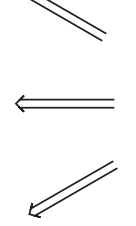

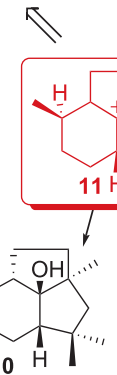

$\pi$
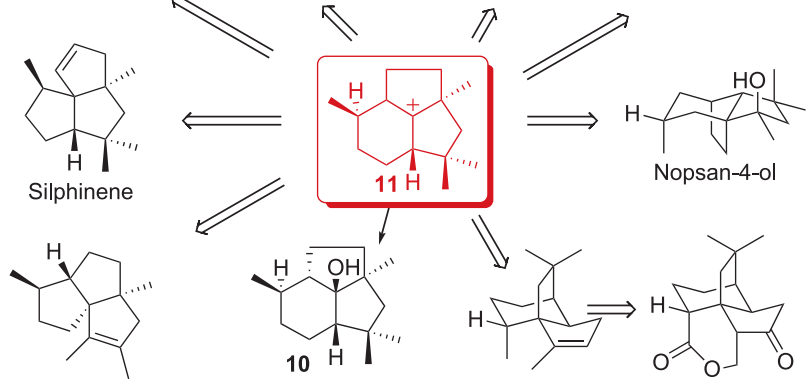

Presilphiperfolan-8 $\beta$-ol
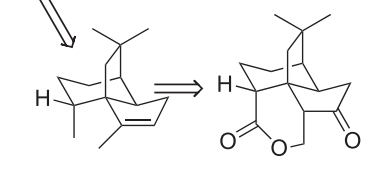

Silphiperfolene

$\alpha$-Terrecyclene

Quadrone

Figure 5. Presilphiperfolan-8-yl cation 11 is the likely precursor of a wide variety of triquinane and related sesquiterpenes from fungi and higher plants.

its presilphiperfolan-9-ol isomer are, however, known compounds, having previously been isolated from the plants Eriophyllum staechadifolium and Fluorensia heterolepsis (21). Interestingly, both the enzymatically generated fungal sesquiterpene alcohol and the plantderived presilphiperfolan- $8 \beta$-ol (10) have the same absolute configuration, as confirmed by direct chiral GC-MS comparison.

Presilphiperfolan-8-yl Cation 11 Plays a Central Role in the Biosynthesis of Numerous Polycyclic

Sesquiterpenes. This report describes the expression and biochemical characterization of the first presilphiperfolan-8 $\beta$-ol synthase obtained from any fungal or plant source. Enhancing the significance of our findings, the presilphiperfolan-8-yl cation $\mathbf{1 1}$ is in fact thought to play a central role in the formation of a wide range of polycyclic sesquiterpenes of both plant and fungal origin (Figure 5). For example, it has been shown that acid-catalyzed solvolysis of presilphiperfolan- $8 \beta$-ol (10) results in rearrangement to the tricyclic sesquiterpene terrecyclene, the precursor of the Aspergillus terreus antibiotic quadrone $(24,25)$. There is also strong experimental evidence supporting the conversion of $\mathbf{1 1}$ to a variety of plant-derived triquinane sesquiterpenes, including silphinenes, modhephene, and isocomene, as well as several other tricyclic sesquiterpenes (24-29) (Figure 5). It is therefore likely that close protein homologues of the fungal presilphiperfolan- $8 \beta$-ol synthase will be found in numerous plant and fungal species that are responsible for the biosynthesis of a wide range of triquinane and related sesquiterpene metabolites.

\section{METHODS}

Fungal Strains and Culture Conditions. Strain B05-10 of Botrytis cinerea Pers.: Fr. [Botryotinia fuckeliana (de Bary) Whetz] is derived from a Vitis field isolate (30). The B05-10 bcg1s mutant obtained by inactivation of the gene encoding the $\mathrm{G} \alpha$ protein BCG1 (19) was provided by B. Tudzynski (University of Münster, Germany). The T4 wild-type strain was isolated from infected tomatoes (Lycopersicum esculentum) grown in a greenhouse in Avignon, France (31). The T4 bcbot1 $\Delta$ mutant is described in our previous study (11). The recipient strains B05-10 $k u 70 \Delta$ and T4 ku80D were recently developed to increase gene deletion efficiency (16). Standard procedures for culture and maintenance of $B$. cinerea wild-type strain and mutants were carried out on NY medium ( $2 \mathrm{~g}$ of malt extract, $2 \mathrm{~g}$ of yeast extract, $15 \mathrm{~g}$ of agar per liter of water) at $21{ }^{\circ} \mathrm{C}$ with $16 \mathrm{~h}$ daylight per $24 \mathrm{~h}$. Growth and conidiation rates were estimated on cultures on NY and on Vegetables-8 (32) solid media. Conidia were counted using microscopy and a Malassez chamber.

Standard Molecular Methods. Genomic DNA was extracted from fungal mycelium using a Sarcosyl-based protocol (33). Gel electrophoresis, restriction enzyme digestion, and Southern blot experiments were performed using standard protocols (34). DNA probes were labeled by the random primer method using the Q-Biogen Nonaprimer Kit and $20 \mu \mathrm{Ci}$ of $\alpha{ }^{32} \mathrm{P}$-dCTP as previously described (31).

Quantitative RT-PCR Analysis. Conidia from the wild-type B. cinerea $\mathrm{B} 05-10 \mathrm{WT}$ and from the $b c g 1 \Delta$ mutant were germinated for $62 \mathrm{~h}$ at $21^{\circ} \mathrm{C}$ in $100 \mathrm{~mL}$ of liquid medium $(2 \mathrm{~g}$ of yeast extract, $10 \mathrm{~g}$ of glucose, $2 \mathrm{~g}$ of $\mathrm{KH}_{2} \mathrm{PO}_{4}, 1.5 \mathrm{~g}$ of $\mathrm{K}_{2} \mathrm{HPO}_{4}, 1 \mathrm{~g}$ of $\left(\mathrm{NH}_{4}\right)_{2} \mathrm{SO}_{4}, 0.5 \mathrm{~g}$ of $\mathrm{MgSO}_{4} \cdot 7 \mathrm{H}_{2} \mathrm{O}$ per liter of water) rotated at 
$120 \mathrm{rpm}$. Then mycelia were quickly filtered and transferred into $100 \mathrm{~mL}$ of the same medium minus the nitrogen sources. After $4 \mathrm{~h}$ at $120 \mathrm{rpm}, 100 \mu \mathrm{L}$ of cyclosporin A (CsA; Calbiochem) suspended in $99 \%$ ethanol/1\% DMSO was added to obtain final concentrations of 0 or $10 \mu \mathrm{g} \mathrm{CsA} \mathrm{mL}{ }^{-1}$. After $3 \mathrm{~h}$ at $120 \mathrm{rpm}$, samples were filtered, washed with sterile water and frozen at $-80^{\circ} \mathrm{C}$. RNA was extracted from fungal tissues with Extract-All reagent (Eurobio).

Primer pairs were designed to amplify the individual $B C B O T 1$ to $B C B O T 5$ genes (NCBI accession number AY277723; Figure 3, panel a) and the constitutively expressed elongation factor gene EF1b (35) (Supplementary Table 1). These specific primers were defined in the 3' region of each gene with the Primer Express 3.0 program according to the supplier's recommendations (ABI) and purchased from Eurogentec ( $40 \mathrm{nmol}$, SePOP purification). Primers were designed to span intron splice sites (BD1 from the $B C B O T 4$ primer pair, $\mathrm{BD} 17$ from the $B C B O T 2$ primer pair) or flanking intron (BCBOT1, $B C B O T 3$, and $E F 1 b$ primer pairs) to prevent or identify amplification of contaminating genomic DNA. This strategy was not optimal for the design of the primer pair for the $B C B O T 5$ gene. Efficiency tests were carried out with a range of four cDNA dilutions (1/10) of wild-type B. cinerea B05-10 cultivated under standard conditions. All gene-specific primer pairs achieved an efficiency between $90 \%$ and $100 \%$, and no primer dimers were observed for any pairs.

RT-PCR assays were carried out using a qPCR ABI Prism SDS 9700 thermocycler including the associated software program SDS 2.2.2 from Applied Biosystems. All reactions were set up in 96-well reaction plates. Each $25-\mu \mathrm{L}$ reaction was performed in the MastermixPlus kit (RT-SN2X-03) from Eurogentec containing the SYBRGreen I dye as a fluorophor. Forward and reverse primer pair concentrations of $200 \mathrm{nM}$ each were chosen for all genes. Negative control reactions without CDNA template were included. Each reaction was carried out in duplicate for efficiency experiments and in triplicate for quantification experiments. PCR cycling parameters were $95^{\circ} \mathrm{C}$ for $10 \mathrm{~min}$, followed by 40 cycles to $95^{\circ} \mathrm{C}$ for $15 \mathrm{~s}, 60^{\circ} \mathrm{C}$ for 1 min with the 9600 emulation protocol. For each experiment, melting curves were acquired by cooling to $60^{\circ} \mathrm{C}$ for $30 \mathrm{~s}$, followed by ramping up the temperature to $95{ }^{\circ} \mathrm{C}$ for $1 \mathrm{~min}$ with a ramp of $2 \%$. Fluorescence data were continuously collected during the ramping from 60 to $95^{\circ} \mathrm{C}$.

Relative quantification of gene expression was achieved using the comparative $\mathrm{Ct}$ method with the EF1b gene as a control for constitutive expression (35). The expression levels of each of the five $B C L$ genes from the B05-10 WT strain grown without CsA were used for calibration with a defined value of 1 . The $\mathrm{Ct}$ value for each gene was measured and the expression level of the genes in the different samples (B05-10, B05-10 + CsA, and $b c g 1 \Delta)$ was calculated by the formula $2^{(-\Delta \Delta c t)}$, representing the $x$-fold difference from the calibration reference. The relative expression software tool included in the SDS 2.2.2 software was used to test whether the expression differences were significant with an interval confidence of $95 \%$.

Gene Inactivation and Complementation by Protoplast

Transformation. The T4 genomic fragment containing the BCBOT2 gene and promoter was PCR-amplified with DyNAzyme DNA polymerase (Finnzymes) by using the upstream primer Cyc1 (Supplementary Table 2) and the downstream primer Cyc2. The resulting 2648-bp PCR fragment was cloned into the pGEMt vector (Promega), yielding $\mathrm{pBcBOT} 2$ plasmid. The bialaphos resistance gene BAR from pCB1265 (36) was introduced into pBcBOT2 at an Eam 1104/ restriction site created using the Seamless cloning kit (Stratagene). To remove the central portion of the BCBOT2 gene, an initial round of PCR amplification was carried out using the primers CycA-seam and CycB-seam that both contain a unique Eam1104/ recognition site. A second PCR with the primers bar-seam-up and bar-seam-low, each containing an Eam $1104 /$ site, allowed amplification of the BAR gene. Both PCR products were digested by Eam 1104 I and then ligated together. In the resulting $\mathrm{pBCBOT} 2 \Delta$, the BAR gene is located between the codons for amino acids 65 and 248 of the BcBOT2 protein.

The $B C B O T 2$ complementation vector was constructed by introducing the Nat1 nourseothricin resistance gene into pBcBOT2. The Nat1 gene and OliC promoter were first PCRamplified from pNR1 vector (kindly provided by B. Tudzynski, University of Munster, Germany) using the primers Nat1-F-NN and Nat1-R-SS that contain Nco I and Sac II sites, respectively (Supplementary Table 2). The PCR product and $p B C B O T 2$ were each digested by these two enzymes and ligated together to obtain the $p B C B O T 2-N a t 1$ complementation vector.

Protoplasts from $B$. cinerea wild-type strains, $k u$ recipient strains, and B05-10 ku70 b bbot $2 \Delta-6$ mutant were prepared and transformed as described previously (31). DNA-mediated transformation was carried out using $2 \mu \mathrm{g}$ of linearized

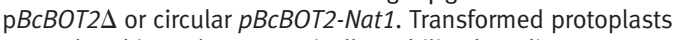
were plated in molten osmotically stabilized medium agar containing $100 \mu \mathrm{g} \mathrm{mL}^{-1}$ bialaphos (glufosinate ammonium, Dr. Ehrenstorfer $\mathrm{GmbH}$, Augsburg, Germany) or $70 \mu \mathrm{g} \mathrm{mL}^{-1}$ nourseothricin (Werner). Transformants were selected after 6-8 days at $23^{\circ} \mathrm{C}$ and subcultured twice on selective media and single spore cultures were prepared to obtain genetically pure transformants. The screening for inactivation of the $B c B O T 2$ gene was carried out by PCR using the Cyc5 primer located upstream of Cyc1 and the Bar547 primer located inside the bar gene. Gene inactivation and complementation were verified by Southern blot hybridization.

Metabolite Analysis. For analysis of metabolite production, strains were grown on malt agar medium (20 g of D-glucose, $10 \mathrm{~g}$ of malt extract, $20 \mathrm{~g}$ of agar, $\mathrm{pH} 6.5-7$ per liter of water) at $25^{\circ} \mathrm{C}$ and used to inoculate Roux bottles or Erlenmeyer flasks. All studied strains were fermented in both surface and shaken cultures. For surface cultures, mycelia were grown in 1-L Roux bottles containing $150 \mathrm{~mL}$ of modified Czapek-Dox medium (50 $\mathrm{mg}$ of D-glucose, $1 \mathrm{~g}$ of yeast extract, $5 \mathrm{~g}$ of $\mathrm{KH}_{2} \mathrm{PO}_{4}, 2 \mathrm{~g}$ of $\mathrm{NaNO}_{3}, 0.5 \mathrm{~g}$ of $\mathrm{MgSO}_{4} \cdot 7 \mathrm{H}_{2} \mathrm{O}$, and $0.01 \mathrm{~g}$ of $\mathrm{FeSO}_{4} \cdot 7 \mathrm{H}_{2} \mathrm{O}, \mathrm{pH}$ 6.5-7.0 per liter of water) at RT. For shaken cultures, mycelia were grown in Erlenmeyer flasks containing $200 \mathrm{~mL}$ of the same medium agitated on an orbital shaker at $140 \mathrm{rpm}$ at $25^{\circ} \mathrm{C}$. Each Roux bottle or Erlenmeyer flask was inoculated with mycelium on six small slices of agar $(1 \mathrm{~cm})$. After 7 days of incubation under fluorescent light, the culture medium was filtered, saturated with $\mathrm{NaCl}$, extracted with ethyl acetate $(3 \times 0.5$ vol $)$ and washed with water $(3 \times 0.25 \mathrm{vol})$. The organic extracts were dried over $\mathrm{Na}_{2} \mathrm{SO}_{4}$ and concentrated to dryness.

${ }^{1} \mathrm{H}$ and ${ }^{13} \mathrm{C}$ NMR measurements on metabolites isolated from culture extracts were obtained on Varian Unity 400 and Varian Innova 600 NMR spectrometers with $\mathrm{SiMe}_{4}$ as the internal reference. Mass spectra were recorded on a $\mathrm{GC}-\mathrm{MS}$ Thermoquest Voyager spectrometer and a VG Autospec-Q spectrometer. HPLC was performed with a Hitachi/Merck L-6270 apparatus equipped with a UV-vis detector (L 6200) and a differential refractometer detector (RI-71). TLC was performed on Merck Kiesegel $60 \mathrm{~F}_{254}, 0.2 \mathrm{~mm}$ thick. Silica gel (Merck) was used for column chromatography. HPLC purification was accomplished with a silica gel column (Hibar 60, $7 \mathrm{~m}, 1 \mathrm{~cm}$ wide, $25 \mathrm{~cm}$ long). Chemicals were products of Fluka or Aldrich. All solvents were freshly distilled.

For metabolite isolation, the culture filtrate $(3 \mathrm{~L})$ was saturated with $\mathrm{NaCl}$, and the aqueous phase was extracted with ethyl acetate. This extract was washed with $\mathrm{H}_{2} \mathrm{O}$ and then dried over anhydrous $\mathrm{Na}_{2} \mathrm{SO}_{4}$. Evaporation of the solvent at reduced pressure gave a yellow oil that was separated by means of column chromatography on silica gel, with a mixture of ethyl ac- 
etate/petroleum ether $(10 \%, 20 \%, 40 \%, 60 \%, 80 \%$, and $100 \%$ ethyl acetate) and $20 \%$ methanol in ethyl acetate as solvent. Extensive spectroscopic analysis by ${ }^{1} \mathrm{H}$ NMR and ${ }^{13} \mathrm{C}$ NMR was used to detect the presence of the various toxins in each fraction. Candidate fractions were further purified by HPLC with an increasing gradient of ethyl acetate to petroleum ether. The toxin structures were analyzed by spectroscopic methods and direct comparison with authentic samples, previously isolated from strains of $B$. cinerea (37). Semipreparative HPLC afforded compounds $1-7$, from both wild-type strains and mutants of B. cinerea (Table 1 and Figure 1).

Infection Assays. The infection of bean (Vicia fabae), tomato (Lycopersicum esculentum), and grape berries (Vitis vinifera) by $B$. cinerea was studied by inoculating detached leaves or fruit with young unsporulated mycelium or conidial suspensions from cultures on NY medium. Bean plants (Caruso cultivar) and tomato plants (Moneymaker cultivar) were grown under greenhouse conditions. Leaves were harvested from 3- to 4-week-old plants and placed in a transparent plastic box lined with tissue moistened with sterile water. Leaves were inoculated with $5-\mathrm{mm}$ diameter plugs of 3-day-old mycelium. Alternatively, conidia were collected from 10-day-old plates and suspended in sucrose phosphate buffer ( $10 \mathrm{mM}$ sucrose, $10 \mathrm{mM} \mathrm{KH}_{2} \mathrm{PO}_{4}$ ) to a final concentration of $10^{5}$ conidia mL ${ }^{-1}$. Droplets of $10 \mu \mathrm{L}$ were applied to the leaves and at the top of the grape berries. Storage boxes containing inoculated leaves and berries were incubated in a growth cabinet at $21{ }^{\circ} \mathrm{C}$ with $16 \mathrm{~h}$ of daylight. Disease development on leaves was recorded daily as radial spread from the inoculation point to the lesion margin. Pathogenicity assays on leaves were repeated 3 times using at least 5 leaves per assay.

Expression and Purification of Recombinant BcBOT2 Protein. Plasmid pJ201:10027 harboring a synthetic BCBOT2 gene, constructed using optimized $E$. coli codons (DNA2.0 Inc.), was extracted from E. coli $\mathrm{DH} 10 \mathrm{~B}$ and treated with Ndel and Xhol. The resulting $B C B O T 2$ DNA was then ligated into expression vector pET28a that had been digested with the same enzymes. The resulting plasmid $\mathrm{pET} 28 \mathrm{a}-B C B O T 2$ was transformed into $E$. coli $\mathrm{XL}-1$ Blue competent cells by electroporation, and the insert sequence was verified by resequencing. The $\mathrm{pET} 28 \mathrm{a}-\mathrm{BCBOT} 2 \mathrm{con}-$ struct was then introduced into $E$. coli BL21(DE3) cells by heat shock transformation. The $E$. coli BL21(DE3) cells harboring the pET28a-BcBOT2 construct were grown in Luria-Bertani broth containing $50 \mathrm{mg} \mathrm{L}^{-1}$ kanamycin. Cultures were incubated at $37^{\circ} \mathrm{C}$ with shaking. At an $\mathrm{OD}_{600}$ of 0.7 , IPTG was added to a final concentration of $0.2 \mathrm{mM}$, and the culture was incubated overnight at $18^{\circ} \mathrm{C}$ with shaking. The cells were harvested by centrifugation and suspended in $30 \mathrm{~mL}$ of lysis buffer $(50 \mathrm{mM}$ Tris, $300 \mathrm{mM} \mathrm{NaCl}, 10 \mathrm{mM}$ imidazole, $5 \mathrm{mM} \beta$-mercaptoethanol, $\mathrm{pH}$ 7.0). The suspension was lysed by sonication and centrifuged. The supernatant was bound to Ni-NTA resin $(5 \mathrm{~mL})$ that had been equilibrated with lysis buffer by shaking at $4^{\circ} \mathrm{C}$ for $1 \mathrm{~h}$. The resin slurry was applied to a column and prewashed with buffer containing 10-30 mM imidazole. The protein was eluted with $70 \mathrm{mM}$ imidazole buffer ( $50 \mathrm{mM}$ Tris, $300 \mathrm{mM} \mathrm{NaCl}, 70 \mathrm{mM}$ imidazole, $5 \mathrm{mM} \beta$-mercaptoethanol, $\mathrm{pH}$ 7.0) at a flow rate of

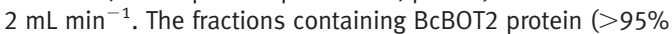
pure by SDS-PAGE, Supplementary Figure 1) were pooled, concentrated by Amicon Ultracel PL-10 centriprep, and exchanged into storage buffer (50 mM PIPES, $20 \%$ (v/v) glycerol, $10 \mathrm{mM}$ $\mathrm{MgCl}_{2}, 100 \mathrm{mM} \mathrm{NaCl}, 5 \mathrm{mM} \beta$-mercaptoethanol, $\mathrm{pH} \mathrm{7.0)}$ with a PD-10 desalting column. The typical yield was $\sim 8.5 \mathrm{mg}$ protein $\mathrm{L}^{-1}$ culture. The protein was flash-frozen in liquid nitrogen and stored at $-80^{\circ} \mathrm{C}$. MALDI-TOF analysis of recombinant $\mathrm{His}_{6}$-tagBcBOT2 protein was carried out on an Applied Biosystems Voyager-DE Pro spectrometer with bovine serum albumin $\left(M_{D}\right.$
66431) added as an internal standard: $M_{D}$ of $47221 \pm 50$ (calcd for $\mathrm{His}_{6}$-tag-BcBOT2 minus $\mathrm{N}$-terminal Met-Gly $M_{\mathrm{D}} 47246$.)

Incubation of BCBOT Protein with FPP and GC-MS Analysis.

Purified recombinant BcBOT2 $(1 \mu \mathrm{M})$ was incubated with $60 \mu \mathrm{M}$ FPP in $4 \mathrm{~mL}$ of assay buffer (50 mM PIPES, $10 \mathrm{mM} \mathrm{MgCl}$, $100 \mathrm{mM} \mathrm{NaCl}, \mathrm{pH} 7.0$ ), overlaid with $4 \mathrm{~mL}$ of HPLC-grade pentane for $1 \mathrm{~h}$ at $30^{\circ} \mathrm{C}$. The reaction mixture was extracted with the $4 \mathrm{~mL}$ pentane overlay followed by $3 \times 4 \mathrm{~mL}$ of HPLC-grade dichloromethane. The combined organic extract was dried over $\mathrm{Na}_{2} \mathrm{SO}_{4}$ and concentrated to $200 \mu \mathrm{L}$ under reduced pressure at $0{ }^{\circ} \mathrm{C}$. A $1-\mu \mathrm{L}$ portion of the concentrated extract was analyzed by capillary GC-MS (Hewlett-Packard Series II gas chromatograph, 5971 series mass selective detector, $70 \mathrm{eV}$, El, positive ion mode; $30 \mathrm{~m} \times 0.25 \mathrm{~mm}$ HP5MS capillary column, using a temperature program of $60-280^{\circ} \mathrm{C}$, a temperature gradient of $20^{\circ} \mathrm{C}$ $\mathrm{min}^{-1}$, and a solvent delay of $4.0 \mathrm{~min}$ ). The incubation gave as major product ( $m / z 222)(-)$-presilphiperfolan-8 $\beta$-ol 10, which was identical in retention time and mass spectrum (Supplementary Figure 2) by direct GC-MS comparison with authentic $\mathbf{1 0}$ that had been isolated from E. staechifolium (gift of Drs. Robert M. Coates and Juan A. Faraldos of the University of Illinois, Urbana, IL). Both the GC-MS of enzymatically generated $\mathbf{1 0}$ and the reference sample of $\mathbf{1 0}$ also exhibited $<5 \%$ of the dehydration product presilphiperfol-7-ene $(\mathrm{m} / \mathrm{z} 204)$, identical to the reference spectrum in the MassFinder database. This dehydration product was possibly generated during the $\mathrm{GC}-\mathrm{MS}$ analysis. Chiral GC-MS analysis (Hydrodex- $\beta-6-T B D M, 25 \mathrm{~m} \times 0.25 \mathrm{~mm}$, using a temperature program of $50-200{ }^{\circ} \mathrm{C}$, a temperature gradient of $10^{\circ} \mathrm{C} \mathrm{min}^{-1}$, and a solvent delay of $4.0 \mathrm{~min}$ ) confirmed that the enzymatic reaction product 10 was a single enantiomer identical to authentic (-)-10.

The same procedure was used for the incubation of $\mathrm{BCBOT2}$ protein with $\left[1,1-{ }^{2} \mathrm{H}_{2}\right]-\mathrm{FPP}$ and $\mathrm{GC}-\mathrm{MS}$ analysis of the resulting [5,5- $\left.{ }^{2} \mathrm{H}_{2}\right]$-presilphiperfolan-8-ol $10(\mathrm{~m} / \mathrm{z} 224)$.

Incubation of BcBOT2 Protein with FPP and with $\left[1,1-{ }^{2} \mathrm{H}_{2}\right]-\mathrm{FPP}$ and NMR Analysis. Purified BcBOT2 protein $(1 \mu \mathrm{M})$ was incubated with FPP $(60 \mu \mathrm{M})$ in $500 \mathrm{~mL}$ of assay buffer, overlaid with $100 \mathrm{~mL}$ of HPLC-grade pentane at $37^{\circ} \mathrm{C}$ for $2 \mathrm{~h}$. The reaction mixture was extracted with an equal volume of HPLC-grade dichloromethane. The combined organic extract was dried over $\mathrm{Na}_{2} \mathrm{SO}_{4}$ and concentrated. The product was purified through a mini- $\mathrm{SiO}_{2}$ gel column $(0.5 \mathrm{~cm} \times 7 \mathrm{~cm}, 2: 1$ pentane/dichloromethane) and concentrated. The ${ }^{1} \mathrm{H}$ NMR spectrum of the purified sample of $\mathbf{1 0}$ was recorded in $\mathrm{C}_{6} \mathrm{D}_{6}$ (Supplementary Figure 3$)$. The same procedure was also carried out using [1,1$\left.{ }^{2} \mathrm{H}_{2}\right]$ FPP $(60 \mu \mathrm{M})$ to give $\left[5,5-{ }^{2} \mathrm{H}_{2}\right]$-presilphiperfolan-8-ol 10 (Supplementary Figures 4 and 5). The original ${ }^{1} \mathrm{H}$ NMR assignments (21) of the $\mathrm{H}-5$ protons of $\mathbf{1 0}$ to the doublets at $\delta 2.25$ and 1.30 were revised and reassigned by ${ }^{1} \mathrm{H}-{ }^{1} \mathrm{H}$ COSY NMR, as well as NOESY, HSQC, and HMBC analysis.

Kinetic Assay of Recombinant BcBOT2. Variable substrate [1- $\left.{ }^{3} \mathrm{H}\right]$-FPP $\left(0.5-35 \mu \mathrm{M} ; 77 \mathrm{Ci} \mathrm{mol}^{-1}\right)$ was incubated with $0.09 \mathrm{nM}$ purified BcBOT2 protein in $1 \mathrm{~mL}$ of assay buffer. The assay mixtures, overlaid with $1.2 \mathrm{~mL}$ of hexanes, were incubated at $30^{\circ} \mathrm{C}$ for $20 \mathrm{~min}$. The reactions were terminated by the addition of $75 \mu \mathrm{L}$ of $500 \mathrm{mM}$ EDTA (pH 8.0) followed by immediate vortexing for $30 \mathrm{~s}$. The organic layer was loaded onto a mini $\mathrm{SiO}_{2}$ gel column $(2 \mathrm{~cm})$ in a Pasteur pipet and expelled with a stream of nitrogen into a scintillation vial containing $7 \mathrm{~mL}$ of OptiFluor. The samples were extracted further with two $1-\mathrm{mL}$ portions of diethyl ether, and the extracts were applied to the same mini column, which was further washed with $750 \mu \mathrm{L}$ of diethyl ether. The tritium content of the combined extracts was determined by liquid scintillation counting. Kinetic constants were determined by using Kaleidagraph software and fitting the data to the Michaelis - Menten equation by nonlinear least-squares regression, which gave the values of $K_{\mathrm{m}}=6.04 \pm 0.98 \mu \mathrm{M}, k_{\text {cat }}=$ 
$3.23 \pm 0.15 \mathrm{~s}^{-1}$ (Supplementary Figure 6). Reported standard deviations in the steady-state kinetic parameters represent the calculated statistical errors in the nonlinear, least-squares regression analysis.

Acknowledgment: This research was supported by grants from MCYT, AGL2006-13401-C02-01/A (Spain), an INRA Jeune Equipe grant (France), and U.S. NIH Grant GM30301 to D.E.C. We are grateful to Anne Witkowski and Guillaume Robin for technical assistance and to Prof. Jason K. Sello for critical comments on the manuscript.

Supporting Information Available: This material is free of charge via the Internet.

\section{REFERENCES}

1. Calvo, A. M., Wilson, R. A., Bok, J. W., and Keller, N. P. (2002) Relationship between secondary metabolism and fungal development, Microbiol. Mol. Biol. Rev. 66, 447-459.

2. Desjardins, A. E., Hohn, T. M., and McCormick, S. P. (1993) Trichothecene biosynthesis in Fusarium species-chemistry, genetics, and significance, Microbiol. Rev. 57, 595-604.

3. Yu, J. H., and Keller, N. P. (2005) Regulation of secondary metabolism in filamentous fungi, Annu. Rev. Phytopathol. 43, 437-458.

4. Williamson, B., Tudzynski, B., Tudzynski, P., and van Kan, J. A. (2007) Botrytis cinerea: the cause of grey mould disease, Mol. Plant Pathol. 8, 561-580.

5. Choquer, M., Fournier, E., Kunz, C., Levis, C., Pradier, J. M., Simon, A., and Viaud, M. (2007) Botrytis cinerea virulence factors: new insights into a necrotrophic and polyphageous pathogen, FEMS Microbiol. Lett. 277, 1-10.

6. Ribéreau-Gayon, J., Ribéreau-Gayon, P., and Seguin, G. (1980) Botrytis in enology, in The Biology of Botrytis (Coley-Smith, J. R., Verhoeff, K., and Jarvis, W. R., Eds.), pp 251-274, Academic Press, London.

7. Collado, I. G., Sanchez, A. J., and Hanson, J. R. (2007) Fungal terpene metabolites: biosynthetic relationships and the control of the phytopathogenic fungus Botrytis cinerea, Nat. Prod. Rep. 24, 674-686.

8. Colmenares, A. J., Aleu, J., Duran-Patron, R., Collado, I. G., and Hernandez-Galan, R. (2002) The putative role of botrydial and related metabolites in the infection mechanism of Botrytis cinerea, J. Chem. Ecol. 28, 997-1005.

9. Deighton, N., Muckenschnabel, I., Colmenares, A. J., Collado, I. G., and Williamson, B. (2001) Botrydial is produced in plant tissues infected by Botrytis cinerea, Phytochemistry 57, 689-692.

10. Tani, H., Koshino, H., Sakuno, E., Cutler, H. G., and Nakajima, H. (2006) Botcinins $E$ and $F$ and botcinolide from Botrytis cinerea and structural revision of botcinolides, J. Nat. Prod. 69, 722-725.

11. Siewers, V., Viaud, M., Jimenez-Teja, D., Collado, I. G., Gronover, C. S., Pradier, J. M., Tudzynski, B., and Tudzynski, P. (2005) Functional analysis of the cytochrome P450 monooxygenase gene bcbot1 of Botrytis cinerea indicates that botrydial is a strainspecific virulence factor, Mol. Plant-Microbe Interact. 18, 602-612.

12. Fillinger, S., Amselem, J., Artiguenave, F., Billault, A., Choquer, M., Couloux, A., Cuomo, C., Dickman, M., Fournier, E., Gioti, A., Giraud, C., Kodira, C., Kohn, L., Legeai, F., Levis, C., Mauceli, E., Pommier, C., Pradier, J. M., Quévillon, E., Rollins, J., Ségurens, B., Simon, A., Viaud, M., Weissenbach, J., Wincker, P., and Lebrun, M. H. (2007) The genome projects of the plant pathogenic fungi Botrytis cinerea and Sclerotinia sclerotiorum, in Macromolecules of Grape and Wines (leandet, P., Clement, C., and Conreux, A., Eds.) TEC and DOC eds., Lavoisier, Paris.
13. Viaud, M., Legeai, F., Pradier, J. M., Brygoo, Y., Bitton, F., Weissenbach, J., Brunet-Simon, A., Duclert, A., Fillinger, S., Fortini, D., Gioti, A., Giraud, C., Halary, S., Lebrun, I., Le Pecheur, P., Samson, D., and Levis, C. (2005) Expressed sequence tags from the phytopathogenic fungus Botrytis cinerea, Eur. J. Plant Pathol. 111, 139-146.

14. Christianson, D. W. (2006) Structural biology and chemistry of the terpenoid cyclases, Chem. Rev. 106, 3412-3442.

15. Ninomiya, Y., Suzuki, K., Ishii, C., and Inoue, H. (2004) Highly efficient gene replacements in Neurospora strains deficient for nonhomologous end-joining, Proc. Natl. Acad. Sci. U.S.A. 101, $12248-$ 12253.

16. Choquer, M., Robin, G., Le Pecheur, P., Giraud, C, Levis, C., and Viaud, M. (2008) Ku70 or Ku80 deficiencies in the fungus Botrytis cinerea facilitate targeting of genes that are hard to knockout in a wild-type context, FEMS Microbiol. Lett. DOI: 10.1111/j.15746968.2008.01388.x.

17. Viaud, M., Brunet-Simon, A., Brygoo, Y., Pradier, J. M., and Levis, C. (2003) Cyclophilin A and calcineurin functions investigated by gene inactivation, cyclosporin A inhibition and cDNA arrays approaches in the phytopathogenic fungus, Botrytis cinerea, Mol. Microbiol. 50, 1451-1465.

18. Schumacher, J., Viaud, M., Simon, A., and Tudzynski, B. (2008) The Galpha subunit BCG1, the phospholipase C (BCPLC1) and the calcineurin phosphatase co-ordinately regulate gene expression in the grey mould fungus Botrytis cinerea, Mol. Microbiol. 67, 10271050.

19. Schulze Gronover, C. S., Kasulke, D., Tudzynski, P., and Tudzynski, B. (2001) The role of $G$ protein alpha subunits in the infection process of the gray mold fungus Botrytis cinerea, Mol. Plant-Microbe Interact. 14, 1293-1302.

20. Schumacher, J., de Larrinoa, I. F., and Tudzynski, B. (2008) Calcineurin-responsive zinc finger transcription factor CRZ1 of Botrytis cinerea is required for growth, development, and full virulence on bean plants, Eukaryotic Cell 7, 584-601.

21. Bohlmann, F., Zdero, C., Jakupovic, J., Robinson, H., and King, R. M. (1981) Eriolanolides, eudesmanolides and a rearranged sesquiterpene from Eriophyllum species, Phytochemistry 20, 2239.

22. Cane, D. E. (1999) Sesquiterpene biosynthesis: cyclization mechanisms, in Comprehensive Natural Products Chemistry. Isoprenoids Including Carotenoids and Steroids (Cane, D. E., Ed.), pp 155-200, Elsevier, Oxford.

23. Wang, S. C., and Tantillo, D. J. (2008) Prediction of a new pathway to presilphiperfolanol, Org. Lett. 10, 4827-4830.

24. Coates, R. M., Ho, J. Z., Klobus, M., and Zhu, L. (1998) Carbocationic rearrangements of silphinane derivatives, J. Org. Chem. 63, 9166-9176.

25. Coates, R. M., Ho, Z., Klobus, M., and Wilson, S. R. (1996) Stereochemistry and reactions of presilphiperfolanol: a branch point marker in triquinane sesquiterpene biogenesis, J. Am. Chem. Soc. 118, 9249-9254.

26. Fitjer, L., Majewski, M., and Monzó-Oltra, H. (1995) Synthesis of tricyclopentanoid sesquiterpenes via rearrangement routes: $( \pm)$ modhephene, $( \pm)$-epimodhephene and $( \pm)$-isocomene, Tetrahedron 51, 8835-8852.

27. Bohlmann, F., and Jakupovic, J. (1980) Neue SesquiterpenKohlenwasserstoffe mit anomalen Kohlenstoffgerüst aus Silphiumarten, Phytochemistry 19, 259-265.

28. Davis, C. E., Duffy, B. C., and Coates, R. M. (2003) Total synthesis of $( \pm)$-cameroonan-7 $\alpha$-ol and biomimetic rearrangements to related nopsane sesquiterpenes, J. Org. Chem. 68, 6935-6943.

29. Weyerstahl, P., Marschal, H., Seelmann, I., and Jakupovic, J. (1998) Cameroonane, prenopsane and nopsane, three new tricyclic sesquiterpene skeletons, Eur. J. Org. Chem. 1205-1212.

30. Quidde, T., Buttner, P., and Tudzynski, P. (1999) Evidence for three different specific saponin-detoxifying activities in Botrytis cinerea and cloning of a gene coding for a putative avecinase, Eur. J. Plant Pathol. 105, 273-283. 
31. Levis, C., Fortini, D., and Brygoo, Y. (1997) Transformation of Botrytis cinerea with the nitrate reductase gene (niaD) shows a high frequency of homologous recombination, Curr. Genet. 32, 157-162.

32. Ansan-Melayah, D., Balesdent, M. H., Buée, M., and Rouxel, T. (1995) Genetic characterization of AvrLm1, the first avirulence gene of Leptosphaeria maculans, Phytopathology 85, 1525-1529.

33. Dellaporta, S. L., Wood, J., and Hicks, J. B. (1983) A plant DNA minipreparation: version 2, Plant Mol. Biol. Rep. 1, 19-21.

34. Sambrook, J., Fritsch, E. F., and Maniatis, T. (1989) Molecular Cloning, A Laboratory Manual, 2nd ed., Cold Spring Harbor Laboratory Press, Cold Spring Harbor, NY.

35. Gioti, A., Simon, A., Le Pecheur, P., Giraud, C., Pradier, J. M., Viaud, M., and Levis, C. (2006) Expression profiling of Botrytis cinerea genes identifies three patterns of up-regulation in planta and an FKBP12 protein affecting pathogenicity, J. Mol. Biol. 358, 372-386.

36. Sweigard, J., Chumley, F., Caroll, A., Farrall, L., and Valent, B. (1997) A series of vectors for fungal transformation, Fungal Genet. Newsletter 44, 52-53.

37. Collado, I. G., Aleu, J., Hernandez-Galan, R., and Duran-Patron, R. (2000) Botrytis species: an intriguing source of metabolites with wide ranges of biological activities. Structure, chemistry and bioactivity of metabolites isolated from Botrytis species, Curr. Org. Chem. 4, 1261-1286. 